\title{
Stable interface structures of heterovalent semiconductor superlattices: the case of $(\mathrm{GaSb})_{n}(\mathrm{ZnTe})_{n}$
}

\author{
Hui-Xiong Deng, ${ }^{1}$ Bing Huang, ${ }^{2}$ and Su-Huai $\mathrm{Wei}^{2}{ }^{2 *}$ \\ ${ }^{1}$ State Key Laboratory for Superlattices and Microstructures, \\ Institute of Semiconductors, Chinese Academy of Sciences, \\ P. O. Box 912, Beijing 100083, China \\ ${ }^{2}$ National Renewable Energy Laboratory, Golden, CO 80401, USA
}

\begin{abstract}
Using first-principles total energy calculation and Monte Carlo simulation, as well as lattice harmonic expansion, we have revealed the chemical trends of the stable atomic configurations at the interface of lattice-matched heterovalent superlattices. Using $(\mathrm{GaSb})_{n}(\mathrm{ZnTe})_{n}$ superlattices as an example, we find that the interfacial energy depends not only on the bond energy but also on the Coulomb energy derived from the donor and acceptor wrong bonds. At short-period limit $(n=1)$, the abrupt [111] interface has the lowest energy even though it is polar, whereas for the long-period superlattices, the nonpolar [110] interface has the lowest energy. This finding provides a general guidance on growing stable lattice-matched heterovalent superlattices for optoelectronic device applications.

PACS numbers: 73.21.Cd, 79.60.Jv, 72.80.Ey, 71.15.Nc
\end{abstract}

$\bar{*}$ swei@nrel.gov

1

(C) 2014. This manuscript version is made available under the Elsevier user license http://www.elsevier.com/open-access/userlicense/1.0/ 


\section{Introduction}

Mixing lattice-matched heterovalent semiconductors AX and BY to form alloys $(\mathrm{AX})_{1-x}(\mathrm{BY})_{x}$ or superlattices $(\mathrm{AX})_{n}(\mathrm{BY})_{m}$ [e.g., AX and BY are the III-V and II-VI (or group IV) compounds, respectively] have attracted much attention recently due to their great potential for novel technology applications because, unlike isovalent semiconductor alloys, their material properties can vary in a wide range as a function of composition $x$ without significant change in the lattice parameters[1-9]. For example, ZnTe/GaSb double-heterostructured materials have been grown using molecular beam epitaxy (MBE) for laser diode applications[10-12]; GaP films have been grown on Si (100) substrates by metal-organic chemical vapour deposition (MOVCD) for possible photovoltaic applications[13-15]; and ZnSe/GaAs heterostructures was prepared as high quality green light emitters[16, 17]. In addition, ZnTe/GaSb also has great potential for tandem solar cell absorbers because GaSb has a direct band gap of $0.8 \mathrm{eV}$ and ZnTe has a direct band gap of $2.4 \mathrm{eV}[18]$; therefore, their alloys and superlattices are capable of covering a large range of the solar spectrum without significant change in the lattice constant. However, unlike isovalent alloys that have been extensively studied[19, 20], there are still many fundamental issues that need to be understood for these heterovalent systems. For example, two effects will affect the formation of the heterovalent superlattice interfaces. (i) In conventional common-atom isovalent alloys (e.g., GaAs/GaP or GaAs/AlAs) there exist only two types of bonds that are the same as their binary compounds with the concentration of the bond type uniquely determined by the alloy concentration $x$. However, for heterovalent AX/BY systems, there will inevitably be two additional types of bonds (i.e., A-Y and B-X, hereinafter referred to as wrong bonds) besides the ones that are the same as in the binary compounds (A-X and B-Y, hereinafter referred to as right bonds), and the number of the wrong bonds and right bonds is not uniquely determined by the alloy concentration $x$. In principle, the wrong bonds will increase the alloy formation energy $[9,21,22]$. Consequently, it is expected that the fewer wrong bonds in the alloy, the more stable for the nonisovalent structures. This suggests that one way to lower the formation energy of heterovalent systems could be forming superlattices with sharp interfaces instead of interfaces with mixed atom interfaces or short-range disordered alloys; (ii) For heterovalent systems, the wrong bonds do not satisfy the two electrons per bond rule. For example, for the III-V/II-VI systems, each II-V wrong bond will have a deficiency 


\section{Computational Methods}

In order to analyze the structural stability at the interfaces of $(\mathrm{AX})_{n}(\mathrm{BY})_{n}$ heterovalent superlattices we select the lattice-matched $(\mathrm{GaSb})_{n} /(\mathrm{ZnTe})_{n}$, superlattices with $n=1-6$ along the [001], [110], and [111] directions, respectively, as model structures. Results for other directions are fitted by using lattice harmonic expansion. For the atomic arrangements parallel to the interface, we choose the $(\sqrt{2} \times \sqrt{2}),(1 \times 1)$, and $(2 \times 2)$ supercells, respectively, for [001], [110], and [111] superlattices. The Monte Carlo simulation is employed to search the stable interfacial structures by swapping the atoms near the interfaces. We only allow a cation swapping with a cation or an anion swapping with an anions in each trial Monte Carlo step. After each swapping, the total energies are calculated using density functional theory (DFT) $[23,24]$ within the generalized gradient approximations (GGA)[25] as implemented in the Vienna ab initio simulation package (VASP) [26-28]. The electron and core interactions are included based on the frozen-core projector-augmented-wave approach[29]. The cutoff energy for the wavefunction expansion is $450 \mathrm{eV}$. For the [001] and [110] orientations, we employed a $8 \times 8 \times 8$ Monkhorst-Pack k-point sampling[30] for the period $n \leq 2,8 \times 8 \times 3$ for period $n=3$ or 4 , and $8 \times 8 \times 1$ for period $n=5$ or 6 superlattices. For the [111] orientation, the according k-point sampling are $6 \times 6 \times 6$ for the period $n \leq 2,6 \times 6 \times 3$ for period $n=3$ or 4 , and $6 \times 6 \times 1$ for period $n=5$ or 6 superlattices, respectively. All these k-point samplings in the Brillouin zone have also been checked to assure the error of interfacial energy is within $0.1 \mathrm{meV} / \AA^{2}$. The GGA calculated lattice constants of bulk GaSb and ZnTe are $6.193 \AA$ 


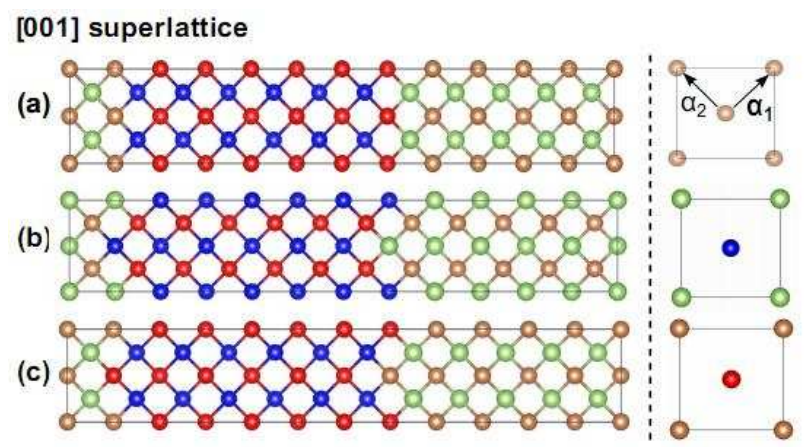

[110] superlattice

(d)

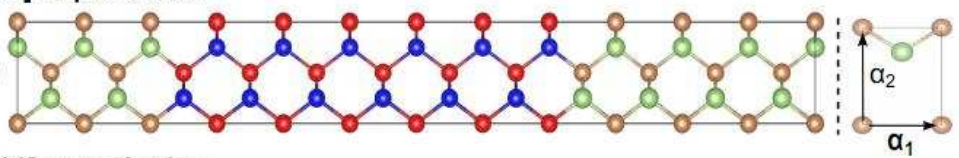

[111] superlattice

(e)

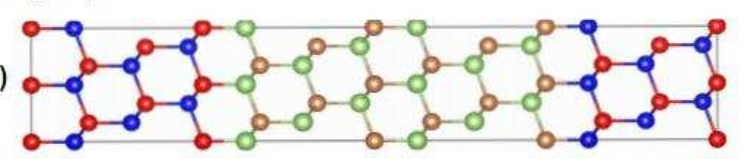

(f)

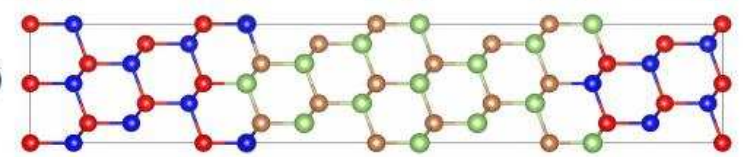

(g)
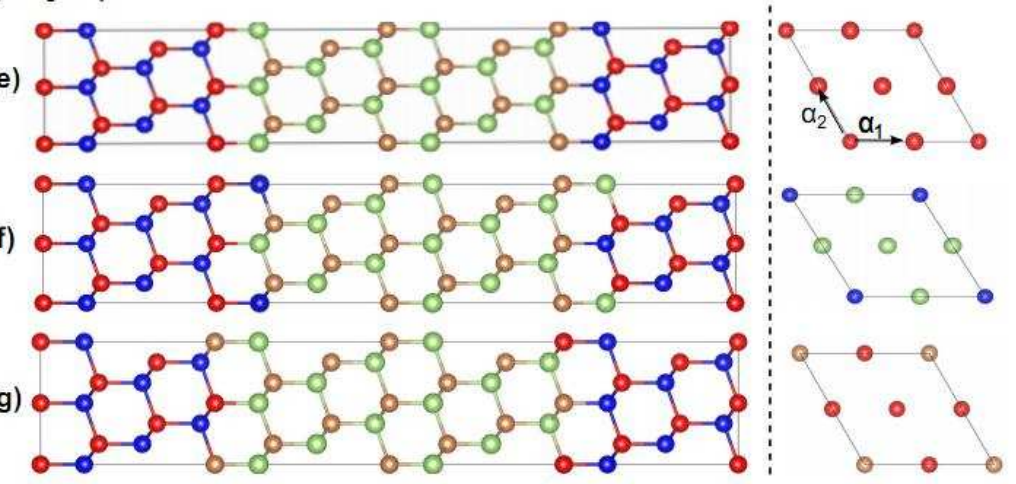

FIG. 1. (Color online) Schematic plot of the structures of lattice-matched heterovalent $(\mathrm{GaSb})_{n} /(\mathrm{ZnTe})_{n}$ superlattices in forming (a) abrupt (AB), (b) mixed anion (MA), and (c) mixed cation (MC) interfaces along the [001] orientation, and (d) AB interfaces along the [110] orientation, and (e) AB, (f) MC, and (g) MA interfaces along the [111] orientation. At the right side of each plot we show the atomic arrangements of the lateral dimensions near one interface. $a_{1}$ and $a_{2}$ are the basis vectors of the lateral plane for the corresponding abrupt interfaces. The green, orange, blue, and red balls indicate the Ga, Sb, Zn, and Te atoms, respectively.

and $6.154 \AA$, respectively, slightly larger than the corresponding experimental values of $6.096 \AA$ and $6.088 \AA$. About 800 Monte Carlo steps are performed for each superlattices to find the minimum total energy. In these processes, to avoid falling into local minimums simulation for each superlattices has been restarted many times with different configurations. Actually because we only consider the atomic configurations in a couple of atomic layers near the interface, the total number of non-equivalent interfacial structures is not quite large. Thus, we have also verified and confirmed the reliability and accuracy of our Monte 
Carlo simulations by comparing to the results of enumerating all the possible non-equivalent interfacial structures for some superlattices (see Appendix A).

The interfacial energy is defined as:

$$
\gamma=\frac{E_{t o t}\left[(A X)_{n}(B Y)_{n}\right]-\left(n E_{t o t}(A X)+n E_{t o t}(B Y)\right)}{2 S}
$$

where $E_{t o t}\left[(A X)_{n}(B Y)_{n}\right], E_{t o t}(A X)$, and $E_{t o t}(B Y)$ are the total energies of superlattices, bulk AX, and BY, respectively. $S$ represents the interfacial area.

\section{Results and Discussions}

To compare the stability of different interface structures, besides the abrupt (AB) interface [Fig. 1(a), 1(d), and 1(e)], we also search the superlattices with low energy nonpolar interfaces, which are expected to be stable, at least for long-period superlattices to avoid polar catastrophe. For the nonpolar interfaces, the interface should have equal donor and acceptor wrong bonds. For the [001] superlattices, our calculations find that the stable nonpolar mixed interfaces are constructed by mixing $50 \% \mathrm{Ga}$ and $50 \% \mathrm{Zn}$ atoms [mixed cation (MC), Fig. 1(b)] or mixing 50\% Sb and 50\% Te atoms [mixed anion (MA), Figs. 1(c)] at the interface, in agreement with previous predictions[31-34]. This is because along the [001] orientations, the number of wrong bonds eliminated and created by swapping atoms are always the same, so the total number of wrong bonds is not changed after the switched of cations or anions at the interfaces. For the [111] superlattices, each time a cation or an anion is switch to remove a wrong bond along the [111] direction, it will simultaneously create three wrong bonds away from the [111] direction with different types (acceptors or donors). Consequently, in order to form nonpolar interfaces only one atom should be switched out of every four [111] wrong bonds, which is consistent with our Monte Carlo simulations [Figs. 1(f) and 1(g)]. Finally, for [110] superlattices, the abrupt interface [Fig. 1(d)] already has an equal number of Ga-Te and Zn-Sb wrong bonds at each interface; therefore, it is already nonpolar.

Our calculated interfacial energies for different orientations and layer thicknesses for the $(\mathrm{GaSb})_{n}(\mathrm{ZnTe})_{n}$ superlattices are shown in Fig. 2. For the nonpolar interface structures (e.g., 50\%-50\% mixed interfaces in the [001] orientation, abrupt interfaces in the [110] orientation, and 25\%-75\% mixed interfaces in the [111] orientation), because the excess electrons 
and holes can locally and fully compensate each other in a single interface, each interface is charge neutral, thus the interfacial energy is not sensitive to the layer thickness of the superlattice. For polar surface, the charge transfer and the Coulomb interaction stabilization is between the adjacent interfaces, which decreases when the layer thickness $n$ increases (Fig. 2), so the interfacial energy increases when $n$ increases. However, for the $n=1$ ultrathin III-V/II-VI superlattices, the Coulomb energy derived from the adjacent interfaces for polar interfaces are similar to that in nonpolar interfaces. In this case, the stability of the interfacial structures should be mainly determined by the interfacial bond energy, which mainly depends on the number of wrong bonds per unit area near the interfaces in these lattice-matched systems. Table I shows that [111] AB interfaces have the lowest number of wrong bonds per unit area, so it has the lowest interfacial energy for $n=1$. Therefore, for the [111] orientation, as discussed above, the polar interfaces are more stable than the nonpolar interfaces when $n$ is small. As $n$ increases, the polar interface becomes more and more unstable. When $n \geq 4$, the interfacial energy of the polar surface is higher than that of the nonpolar interfaces. For the [110] orientation the AB interfaces are already nonpolar, so the nonpolar surface is always more stable. For the [001] orientation, because the formation of mixed nonpolar interfaces doesn't change the number of wrong bonds per unit compared with polar interfaces (Table I), the dominant factor for the interface stability is the Coulomb energy. Because the mixed nonpolar interfaces always have the most negative Coulomb energy, the [001] orientation nonpolar interfaces are also always energetically more favorable than the polar interfaces.

Our calculated results show that in the short-period limit $(n=1)$, the [111] orientation has the lowest interfacial energy of $2.7 \mathrm{meV} / \AA^{2}$ between the three principal orientations. As discussed above, this is because in the $n=1$ ultrathin superlattices the stability of the interface is controlled by the number of wrong bonds per unit area. For the [001], [110], and [111] orientations, they are $4 / a^{2}, 4 / \sqrt{2} a^{2}$ and $4 / \sqrt{3} a^{2}$ (Table I), respectively. Therefore, the interfacial energy has the order of $\gamma_{001}>\gamma_{110}>\gamma_{111}$. For larger $n$, the Coulomb interaction becomes a dominant factor, so all the interfaces become nonpolar. For the nonpolar surfaces, the minimum number of wrong bonds per unit interfacial area are $4 / a^{2}, 4 / \sqrt{2} a^{2}$, and $6 / \sqrt{3} a^{2}$ for the [001], [110] and [111] interfaces, respectively. Therefore, the interfacial energy has the order of $\gamma_{001}>\gamma_{111}>\gamma_{110}$. That is for thick superlattice, the [110] interface has the lowest energy. 
TABLE I. Detailed information for the AB, MC, or MA interfaces of the

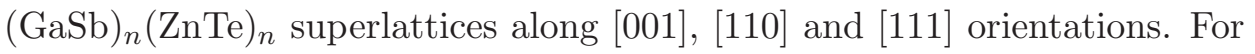
the number of wrong bonds, the one outside and inside the parentheses are for the two interfaces of the superlattices, respectively.

\begin{tabular}{|c|c|c|c|c|c|c|}
\hline Growth orientation & & [001] & {$[110]$} & & {$[111]$} & \\
\hline Interfacial characters & $\mathrm{AB}$ & $\mathrm{MC} \mathrm{MA}$ & $\mathrm{AB}$ & $\mathrm{AB}$ & $\mathrm{MC}$ & MA \\
\hline Polar $(\mathrm{P})$ or nonpolar (NP) & $\mathrm{P}$ & NP NP & NP & $\mathrm{P}$ & NP & NP \\
\hline \multicolumn{7}{|l|}{ Wrong bonds } \\
\hline $\mathrm{Ga}-\mathrm{Te}$ & $4(0)$ & $2(2) 2(2)$ & $1(1)$ & $4(0)$ & $3(3)$ & $3(3)$ \\
\hline $\mathrm{Zn}-\mathrm{Sb}$ & $0(4)$ & $2(2) 2(2)$ & $1(1)$ & $0(4)$ & $3(3)$ & $3(3)$ \\
\hline Total & $4(4)$ & $4(4) 4(4)$ & $2(2)$ & $4(4)$ & $6(6)$ & $6(6)$ \\
\hline $\begin{array}{l}\text { Number of wrong } \\
\text { bonds per unit area }\end{array}$ & $\frac{4}{a^{2}}$ & $\frac{4}{a^{2}} \quad \frac{4}{a^{2}}$ & $\frac{4}{\sqrt{2} a^{2}}$ & $\frac{4}{\sqrt{3} a^{2}}$ & $\frac{6}{\sqrt{3} a^{2}}$ & $\frac{6}{\sqrt{3} a^{2}}$ \\
\hline
\end{tabular}

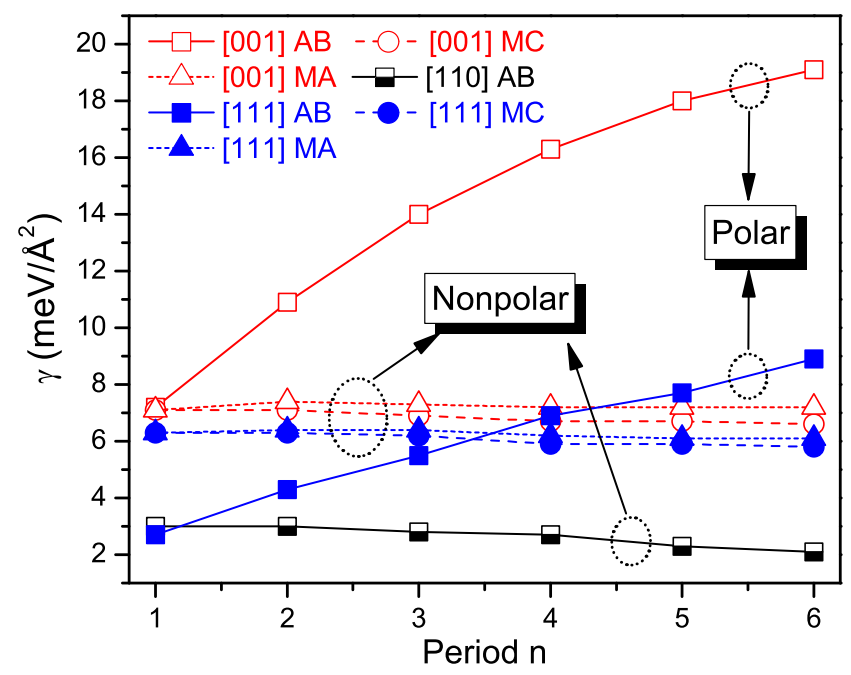

FIG. 2. (Color online) Interfacial energies of [001] (red empty symbols), [110] (black half-filled symbols), and [111] (blue solid symbols) orientations of the $(\mathrm{GaSb})_{n}(\mathrm{ZnTe})_{n}$ superlattices as a function of layer period $n$ in forming polar or nonpolar interfaces.

In order to further confirm the favored growth orientation in the heterovalent superlattices, we calculate the interfacial energy along an arbitrary growth orientation as an function of the polar and azimuthal angles $(\theta, \phi)$. We do this by employing crystal symmetry and 


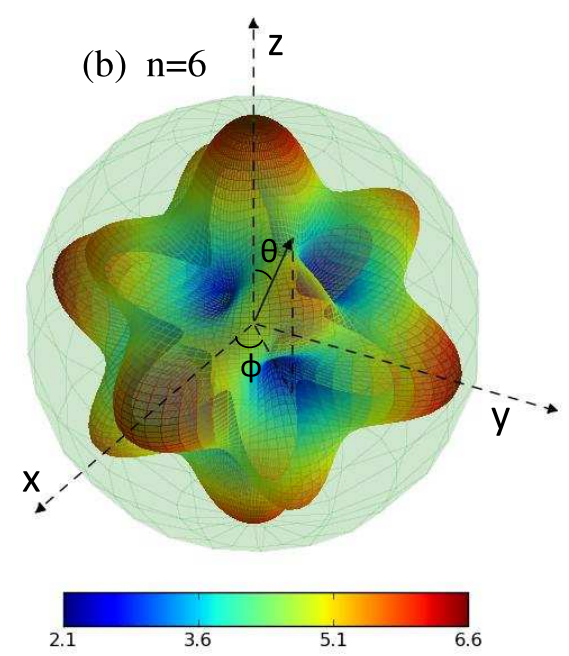

FIG. 3. (Color online) Interfacial energy (in $\left.\mathrm{meV} / \AA^{2}\right)$ of $(\mathrm{GaSb})_{n}(\mathrm{ZnTe})_{n}$ superlattices as a function of orientation for periods (a) $n=1$ and (b) $n=6$.

expanding the interfacial energy for any orientation $[l, m, n]$ or angle $(\theta, \phi)$ in terms of the cubic lattice harmonics with $l_{\max }=6[35,36]$ :

$$
\begin{aligned}
\gamma(\theta, \phi) & =a+b\left[\sin (\theta)^{4} \cos (\phi)^{4}+\sin (\theta)^{4} \sin (\phi)^{4}\right. \\
& \left.+\cos (\theta)^{4}-\frac{3}{5}\right]+c\left\{\sin (\theta)^{4} \cos (\theta)^{2} \cos (\phi)^{2} \sin (\phi)^{2}\right. \\
& +\frac{1}{22}\left[\sin (\theta)^{4} \cos (\phi)^{4}+\sin (\theta)^{4} \sin (\phi)^{4}\right. \\
& \left.\left.+\cos (\theta)^{4}-\frac{3}{5}\right]-\frac{1}{105}\right\}
\end{aligned}
$$

where $\theta \in[0, \pi]$ and $\phi \in[0,2 \pi)$. The parameters a, b, and c can be fitted using the interfacial energies of the $[001](\theta=0),[110](\theta=\pi / 2, \phi=\pi / 4)$ and $[111](\theta=\arccos (1 / \sqrt{3}), \phi=$ $\pi / 4)$ directions. Figure 3 shows the interfacial energy of the $(\mathrm{GaSb})_{n}(\mathrm{ZnTe})_{n}$ superlattices as a function of the orientation for (a) $n=1$ and (b) $n=6$. It is found that whether in the short-period limit or in long-period superlattices, the [001] orientation always has the highest interfacial energy. However, for $n=1$ ultrathin superlattices, the minimum interfacial energy is located at the [111] and equivalent directions. For $n=6$, the [110] and its equivalent orientations have the lowest interfacial energy among all growth orientations. These results confirmed our expectation that for the $n=1$ ultrathin superlattices, the [111] orientations is most preferred, and in long-period superlattices, the [110] orientations have the lowest interfacial energy among all growth orientations. On contrary, for lattice-mismatched isovalent superlattices, the [201] superlattices usually have the lowest energy [20]. 


\section{Acknowledgments}

The work at Institute of Semiconductors, Chinese Academy of Sciences was supported by the National Basic Research Program of China (973 Program) Grant No. G2009CB929300, and the National Natural Science Foundation of China under Grants No. 61121491, and No. 11104264. The work at NREL was supported by the U.S. Department of Energy under Contract No. DE-AC36-08GO28308.

\section{Appendix A}

To test the reliability and rationality of the Monte Carlo simulation, we have also tried to enumerate all the possible non-equivalent interfacial structures for some superlattces. For example, Fig. 4 shows the interfacial energies of $(\mathrm{GaSb})_{6}(\mathrm{ZnTe})_{6}$ superlattices for the enumerative interfacial atomic configurations along the (001), (110) and (111) orientations. In the [001] direction, the structures A, B, C and D have nearly the same minimum interfacial energy and all of them are mixed-cation (MC) or mixed-anion (MA) nonpolar interfaces. Similarly, the more stable structures G, H, M, K, L, I, N, and J in the [111] direction also are MC or MA nonpolar interfaces. In the [110] direction, the nonpolar structure $\mathrm{F}$ has the lowest interfacial energy of $3.15 \mathrm{meV} / \AA^{2}$. These results are consistent with that of the Monte 
Carlo search, suggesting that our Monte Carlo simulations are reasonable and reliable.

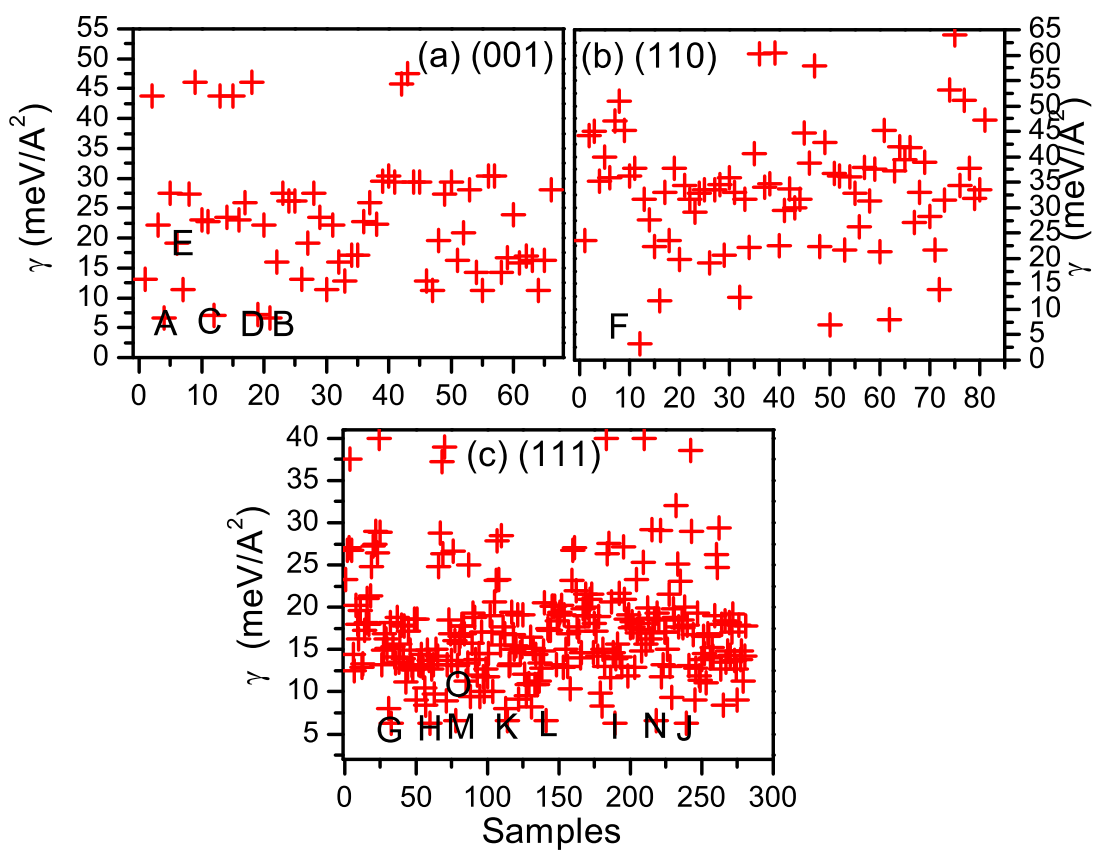

FIG. 4. (Color online) Interfacial energies (in $\mathrm{meV} / \AA^{2}$ ) of GaSb/ZnTe (a) (001), (b) (110) and (c) (111) interfaces calculated for all possible interfacial atomic configurations.

\section{References}

[1] J. R. Lang, J. Faucher, S. Tomasulo, K. Nay Yaung, and M. Larry Lee, Appl. Phys. Lett. 103, 092102 (2013).

[2] O. Romanyuk, T. Hannappel, and F. Grosse, Phys. Rev. B 88, 115312 (2013).

[3] A. Punya and W. R. L. Lambrecht, Phys. Rev. B 88, 075302 (2013).

[4] J.-H. Yang, Y. Zhai, H. Liu, H. Xiang, X. Gong, and S.-H. Wei, J. Am. Chem. Soc. 134, 12653 (2012).

[5] T. Watkins, A. V. G. Chizmeshya, L. Jiang, D. J. Smith, R. T. Beeler, G. Grzybowski, C. D. Poweleit, J. Menndez, and J. Kouvetakis, J. Am. Chem. Soc. 133, 16212 (2011). 
[6] K. Maeda, T. Takata, M. Hara, N. Saito, Y. Inoue, H. Kobayashi, and K. Domen, J. Am. Chem. Soc. 127, 8286 (2005).

[7] A. Colli, E. Pelucchi, and A. Franciosi, Appl. Phys. Lett. 83, 81 (2003).

[8] A. Frey, U. Bass, S. Mahapatra, C. Schumacher, J. Geurts, and K. Brunner, Phys. Rev. B 82, 195318 (2010).

[9] S. Wang and L.-W. Wang, Phys. Rev. B 83, 115208 (2011).

[10] J. Fan, X. Liu, L. Ouyang, R. E. Pimpinella, M. Dobrowolska, J. K. Furdyna, D. J. Smith, and Y.-H. Zhang, J. Vac. Sci. Technol. B 31, 03 (2013).

[11] J. Fan, L. Ouyang, X. Liu, J. Furdyna, D. Smith, and Y.-H. Zhang, J. Cryst. Growth 371, 122 (2013).

[12] J. Fan, X. Liu, J. K. Furdyna, and Y.-H. Zhang, Appl. Phys. Lett. 101, 121909 (2012).

[13] H. Döscher, B. Borkenhagen, G. Lilienkamp, W. Daum, and T. Hannappel, surf. Sci. 605, L38 (2011).

[14] H. Döscher, T. Hannappel, B. Kunert, A. Beyer, K. Volz, and W. Stolz, Appl. Phys. Lett. 93, 172110 (2008).

[15] H. Döscher, B. Kunert, A. Beyer, O. Supplie, K. Volz, W. Stolz, and T. Hannappel, J. Vac. Sci. Technol. B 28, C5H1 (2010).

[16] M. A. Haase, J. Qiu, J. M. DePuydt, and H. Cheng, Appl. Phys. Lett. 59, 1272 (1991).

[17] O. Schulz, M. Strassburg, T. Rissom, S. Rodt, L. Reissmann, U. Pohl, D. Bimberg, M. Klude, D. Hommel, S. Itoh, K. Nakano, and A. Ishibashi, Phys. Status Solidi B 229, 943 (2002).

[18] S. Wang, D. Ding, X. Liu, X.-B. Zhang, D. Smith, J. Furdyna, and Y.-H. Zhang, J. Cryst. Growth 311, 2116 (2009).

[19] C. Woolley, Compound Semiconductors, edited by R. K. Willardson and H. L. Goering (Reinhold, New York, 1962) p. 3.

[20] S.-H. Wei and A. Zunger, Phys. Rev. B 39, 3279 (1989).

[21] S. Wang and L.-W. Wang, Phys. Rev. Lett. 104, 065501 (2010).

[22] M. Peressi, L. Colombo, R. Resta, S. Baroni, and A. Baldereschi, Phys. Rev. B 48, 12047 (1993).

[23] W. Kohn and L. J. Sham, Phys. Rev. 140, A1133 (1965).

[24] P. Hohenberg and W. Kohn, Phys. Rev. 136, B864 (1964).

[25] J. P. Perdew, K. Burke, and M. Ernzerhof, Phys. Rev. Lett. 77, 3865 (1996). 
[26] G. Kresse and J. Hafner, Phys. Rev. B 47, 558 (1993).

[27] G. Kresse and J. Hafner, Phys. Rev. B 48, 13115 (1993).

[28] G. Kresse and J. Furthmuller, Comput. Mater. Sci. 6, 15 (1996).

[29] G. Kresse and D. Joubert, Phys. Rev. B 59, 1758 (1999).

[30] H. J. Monkhorst and J. D. Pack, Phys. Rev. B 13, 5188 (1976).

[31] A. Kley and J. Neugebauer, Phys. Rev. B 50, 8616 (1994).

[32] H. H. Farrell and R. A. LaViolette, J. Vac. Sci. Technol. B 22, 2250 (2004).

[33] H. H. Farrell and R. A. LaViolette, J. Vac. Sci. Technol. B 23, 406 (2005).

[34] G. Bratina, L. Vanzetti, L. Sorba, G. Biasiol, A. Franciosi, M. Peressi, and S. Baroni, Phys. Rev. B 50, 11723 (1994).

[35] F. C. von der Lage and H. A. Bethe, Phys. Rev. 71, 612 (1947).

[36] Y.-H. Li, X. G. Gong, and S.-H. Wei, Appl. Phys. Lett. 88, 042104 (2006). 